\title{
DIFFERENTIAL SIMPLICITY AND CYCLIC MAXIMAL IDEALS OF THE WEYL ALGEBRA $A_{2}(K)$
}

\author{
ADA MARIA DE S. DOERING ${ }^{1}$ \\ Instituto de Matemática, Universidade Federal do Rio Grande do Sul, Avenida \\ Bento Gonçalves 9500, 91509 - 900 Porto Alegre - RS, Brazil \\ YVES LEQUAIN ${ }^{2}$ \\ Instituto de Matemática Pura e Aplicada, Estrada Dona Castorina 110, \\ 22460 - 320 Rio de Janeiro - RJ, Brazil \\ e-mail: ylequain@impa.br \\ and CYDARA C. RIPOLL ${ }^{3}$ \\ Instituto de Matemática, Universidade Federal do Rio Grande do Sul, Avenida \\ Bento Gonçalves 9500, 91509 - 900 Porto Alegre - RS, Brazil \\ e-mail:cydara@mat.ufrgs.br
}

(Received 30 August, 2005; revised 1 March, 2006; accepted 10 March, 2006)

\begin{abstract}
Let $K$ be a field of characteristic zero and $A_{2}:=A_{2}(K)$ the $2 n d-$ Weyl algebra over $K$. We establish a close connection between the maximal left ideals of $A_{2}$ and the simple derivations of $K\left[X_{1}, X_{2}\right]$.

MAIN TheOREM. Let $d=\partial_{1}+\beta \partial_{2}$ be a simple derivation of $K\left[X_{1}, X_{2}\right]$ with $\beta \in$ $K\left[X_{1}, X_{2}\right]$. Then, there exists $\gamma \in K\left[X_{1}, X_{2}\right]$ such that $d+\gamma$ generates a maximal left ideal of $A_{2}$. More precisely, the following is true:

(1) $\operatorname{deg}_{X_{2}}(\beta) \geq 2$ or $\operatorname{deg}_{X_{2}}(\beta)=1$ and $\operatorname{deg}_{X_{1}}\left(\partial_{2}(\beta)\right) \geq 1$;

(2) $d+g X_{2}$ generates a maximal left ideal of $A_{2}$ if $g \in K\left[X_{1}\right] \backslash\{0\}$ is such that

(a) $g \notin-\left(\partial_{2}^{2}(\beta) / 2\right) \mathbb{N}$ when $\operatorname{deg}_{X_{2}}(\beta) \geq 2$,

(b) $\operatorname{deg}_{X_{1}}(g)<\operatorname{deg}_{X_{1}}\left(\partial_{2}(\beta)\right)$ when $\operatorname{deg}_{X_{2}}(\beta)=1$.

As applications, we obtain large families of concrete examples of cyclic maximal left ideals of $A_{2}$; such examples have been rather scarce so far.

2000 Mathematics Subject Classification. Primary 16S32. Secondary 16D25, 16P90, 16W25.
\end{abstract}

1. Introduction. Let $K$ be a field of characteristic zero and $A_{2}:=A_{2}(K)$ the 2 nd Weyl algebra over $K$.

In 1988, Berstein and Lunts showed in [1] that the "generic" differential operator of $A_{2}$ with total degree $m \geq 4$ generates a maximal left ideal of $A_{2}$. This result however

\footnotetext{
${ }^{1}$ This research was partially supported by FAPERGS, Brazil.

2 This research was partially supported by CNPq, Brazil and by a grant from the group ALGAPRONEX/MCT, Brazil.

${ }^{3}$ This research was partially supported by FAPERGS, Brazil.
} 
is not effective and the only concrete known examples of cyclic maximal left ideals of $A_{2}$ are the following:

$$
\begin{aligned}
& A_{2} \cdot\left(\partial_{1}+\left(\lambda X_{1} X_{2}+1\right) \partial_{2}+2 X_{2}\right) \text { with } \lambda \in K \backslash\{0\}, \\
& A_{2} \cdot\left(\partial_{1}+\left(\lambda X_{2}^{2}+X_{1} X_{2}+1\right) \partial_{2}+v X_{2}\right) \text { with } \lambda \in K \backslash \mathbb{Q}, v \in K \text { and } v / \lambda \notin \mathbb{Z},
\end{aligned}
$$

that were given by Stafford in [10] for a field $K$ of infinite degree over $\mathbb{Q}$, and

$$
A_{2} \cdot\left(\partial_{1}+\left(a X_{2}+b\right) \partial_{2}+h X_{2}\right) \text { with } a, b, h \in K\left[X_{1}\right] \backslash\{0\} \text {, and } \operatorname{deg}(a)>\max \{\operatorname{deg}(b),
$$
$\operatorname{deg}(h)\}$,

that was given by Coutinho in [3], and variations of them.

In this paper, we shall establish a close relationship between the cyclic maximal left ideals of $A_{2}$ and the simple derivations of $K\left[X_{1}, X_{2}\right]$.

MAIN TheOREM. Let $d=\partial_{1}+\beta \partial_{2}$ be a simple derivation of $K\left[X_{1}, X_{2}\right]$ with $\beta \in$ $K\left[X_{1}, X_{2}\right]$. Then, there exists $\gamma \in K\left[X_{1}, X_{2}\right]$ such that $d+\gamma$ generates a maximal left ideal of $A_{2} \cdot{ }^{4}$ More precisely, the following is true:

(1) $\operatorname{deg}_{X_{2}}(\beta) \geq 2$ or $\operatorname{deg}_{X_{2}}(\beta)=1$ and $\operatorname{deg}_{X_{1}}\left(\partial_{2}(\beta)\right) \geq 1$;

(2) $d+g X_{2}$ generates a maximal left ideal of $A_{2}$ if $g \in K\left[X_{1}\right] \backslash\{0\}$ is such that

(a) $g \notin-\left(\partial_{2}^{2}(\beta) / 2\right) \mathbb{N}$ when $\operatorname{deg}_{X_{2}}(\beta) \geq 2$,

(b) $\operatorname{deg}_{X_{1}}(g)<\operatorname{deg}_{X_{1}}\left(\partial_{2}(\beta)\right)$ when $\operatorname{deg}_{X_{2}}(\beta)=1$.

Through this connection, we obtain some new insight into maximal left ideals of $A_{2}$. In particular, we obtain a rich source of concrete examples of cyclic maximal left ideals of $A_{2}$ (hence also of concrete irreducible non-holonomic modules over $A_{2}$ ). Besides recovering all the above concrete examples, we easily produce examples of the type $A_{2} \cdot\left(\partial_{1}+\beta \partial_{2}+\gamma\right)$ with $K$ an arbitrary field of characteristic zero, $\beta, \gamma \in$ $K\left[X_{1}, X_{2}\right], \operatorname{deg}_{X_{2}}(\beta)$ equal to an arbitrary integer $\geq 1$ (and not only $\operatorname{deg}_{X_{2}}(\beta)$ equal to 1 or 2 as in the known cases).

Throughout this paper, $K$ will be an arbitrary field of characteristic zero, $X_{1}, X_{2}$ two indeterminates over $K$ and $A_{2}:=A_{2}(K):=K\left[X_{1}, X_{2}\right]\left\langle\partial_{1}, \partial_{2}\right\rangle$ the $2 n d$-Weyl algebra over $K$, where $\partial_{i}$ denotes the derivation $\partial / \partial X_{i}$ of $K\left[X_{1}, X_{2}\right]$. For an element $f \in K\left[X_{1}\right]$, we shall often use $f^{\prime}$ instead of $\partial_{1}(f)$.

Every non-zero element $R$ of $K\left[X_{1}, X_{2}\right]\left\langle\partial_{2}\right\rangle$ may uniquely be written in the form $R=\sum_{i=0}^{r} g_{i} \partial_{2}^{i}$, with $g_{i} \in K\left[X_{1}, X_{2}\right]$ for every $i, g_{r} \neq 0 ; r$ is then said to be the order of $R$. If $d$ is a derivation of $K\left[X_{1}, X_{2}\right]$, if $\gamma \in K\left[X_{1}, X_{2}\right]$ and if $S \in K\left[X_{1}, X_{2}\right]\left\langle\partial_{2}\right\rangle \backslash K$, we say that $S$ is a Darboux operator of $d+\gamma$ if $[d+\gamma, S] \in K\left[X_{1}, X_{2}\right] S$.

If $d$ is a derivation of a $\operatorname{ring} B$, an ideal $I$ of $B$ is said to be a $d$-ideal if $d(I) \subseteq I$. If the only $d$-ideals of $B$ are (0) and (1), $d$ is said to be a simple derivation; we shall also say that $B$ is $d$-simple.

2. Proof of the Main Theorem. One important ingredient in the proof of this theorem will be the following result.

TheOREM 2.1. ([5, Theorem 2.7], [2, Theorem 2.2]) Let $d=\partial_{1}+\beta \partial_{2}$, with $\beta \in$ $K\left[X_{1}, X_{2}\right]$, be a derivation of $K\left[X_{1}, X_{2}\right]$. Let $\gamma \in K\left[X_{1}, X_{2}\right]$. Then the following statements are equivalent:

(i) $A_{2} \cdot(d+\gamma)$ is a maximal left ideal of $A_{2}$.

(ii) $d+\gamma$ does not admit any Darboux operator in $K\left[X_{1}, X_{2}\right]\left\langle\partial_{2}\right\rangle$.

We recall a technical lemma.

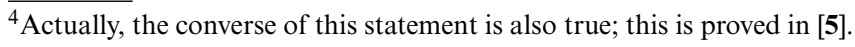


LEMMA 2.2. Let $d=\partial_{1}+\beta \partial_{2}$, with $\beta \in K\left[X_{1}, X_{2}\right]$, be a derivation of $K\left[X_{1}, X_{2}\right]$ and $\gamma \in K\left[X_{1}, X_{2}\right]$. Let $j \geq 0$ be an integer and $f_{j} \in K\left[X_{1}, X_{2}\right]$. Then

$$
\left[d+\gamma, f_{j} \partial_{2}^{j}\right]=\left(d\left(f_{j}\right)-j \partial_{2}(\beta) f_{j}\right) \partial_{2}^{j}-f_{j} \sum_{l=1}^{j}\left(C_{j}^{l+1} \partial_{2}^{l+1}(\beta)+C_{j}^{l} \partial_{2}^{l}(\gamma)\right) \partial_{2}^{j-l} .
$$

Proof. This is an easy computation.

Definition. Let $d=\partial_{1}+\beta \partial_{2}$, with $\beta \in K\left[X_{1}, X_{2}\right]$, be a simple derivation of $K\left[X_{1}, X_{2}\right]$. Let $m \geq 1$ be an integer and $\gamma \in K\left[X_{1}, X_{2}\right]$. We define $P_{m}(Z, \gamma)$ in the following way:

$$
P_{m}(Z, \gamma):=\partial_{1}(Z)+\beta \partial_{2}(Z)+\partial_{2}(\beta) Z-\left(C_{m}^{2} \partial_{2}^{2}(\beta)+C_{m}^{1} \partial_{2}(\gamma)\right) .
$$

Proof of the Main Theorem. (1) Since $d$ is a simple derivation of $K\left[X_{1}, X_{2}\right]$, $\operatorname{deg}_{X_{2}}(\beta) \geq 1$ because, otherwise, letting $\int \beta$ be an element of $K\left[X_{1}\right]$ whose derivative is $\beta$, the ideal $\left(X_{2}-\int \beta\right) K\left[X_{1}, X_{2}\right]$ would be a non-trivial $d$-ideal of $K\left[X_{1}, X_{2}\right]$.

Now, if $\operatorname{deg}_{X_{2}}(\beta)=1$, say $\beta=a X_{2}+b$ with $a, b \in K\left[X_{1}\right], a \neq 0$, then $b \neq 0$ and $\operatorname{deg}(a) \geq 1$. Indeed, if $b$ were equal to zero, then $X_{2} K\left[X_{1}, X_{2}\right]$ would be a non-trivial $d$-ideal of $K\left[X_{1}, X_{2}\right]$. If $a$ belonged to $K \backslash\{0\}$, then taking $h:=-\sum_{i=0}^{r} \partial_{1}^{i}(b) / a^{i+1}$ where $r:=\operatorname{deg}_{X_{1}}(b) \geq 0, h$ would be an element of $K\left[X_{1}\right]$ such that $h^{\prime}=a h+b$, and hence such that $d\left(X_{2}-h\right)=\left(a X_{2}+b\right)-(a h+b)=a\left(X_{2}-h\right)$ and $\left(X_{2}-h\right) K\left[X_{1}, X_{2}\right]$ would be a non-trivial $d$-ideal of $K\left[X_{1}, X_{2}\right]$.

(2) First we establish the following result.

Claim. Let $m \geq 1$ be an integer and $\gamma \in K\left[X_{1}, X_{2}\right]$. If $d+\gamma$ admits a Darboux operator of order $m$ in $K\left[X_{1}, X_{2}\right]\left\langle\partial_{2}\right\rangle$, then the differential equation $P_{m}(Z, \gamma)=0$ has a solution in $K\left[X_{1}, X_{2}\right]$.

Indeed, by hypothesis, there exists an operator $R=\sum_{i=0}^{m} f_{i} \partial_{2}^{i}$ with $f_{i} \in K\left[X_{1}, X_{2}\right]$ for every $i, f_{m} \neq 0$ and $g \in K\left[X_{1}, X_{2}\right]$, such that

$$
[d+\gamma, R]=g R
$$

By Lemma 2.2, we have the following equalities:

$$
\begin{aligned}
\left(\text { coefficient of } \partial_{2}^{m} \text { in }[d+\gamma, R]\right)= & d\left(f_{m}\right)-m \partial_{2}(\beta) f_{m}, \\
\left(\text { coefficient of } \partial_{2}^{m-1} \text { in }[d+\gamma, R]\right)= & \left(d\left(f_{m-1}\right)-(m-1) \partial_{2}(\beta) f_{m-1}\right) \\
& -\left(C_{m}^{2} \partial_{2}^{2}(\beta)+C_{m}^{1} \partial_{2}(\gamma)\right) f_{m} .
\end{aligned}
$$

Evidently, we also have

$$
g R=\sum_{i=0}^{m} g f_{i} \partial_{2}^{i}
$$

Hence, by (2) and (3), we get

$$
d\left(f_{m}\right)=\left(g+m \partial_{2}(\beta)\right) f_{m}
$$

Thus $f_{m} K\left[X_{1}, X_{2}\right]$ is a non-zero $d$-ideal of $K\left[X_{1}, X_{2}\right]$, and therefore

$$
f_{m} \in K \backslash\{0\},
$$


since $K\left[X_{1}, X_{2}\right]$ is $d$-simple. Then $d\left(f_{m}\right)=0$,

$$
g=-m \partial_{2}(\beta)
$$

by (5), and

$$
\text { (coefficient of } \left.\partial_{2}^{m-1} \text { in } g R\right)=-m \partial_{2}(\beta) f_{m-1} \text {. }
$$

Now, looking at the terms in $\partial_{2}^{m-1}$ in (2), we have, by (4) and (7), $d\left(f_{m-1}\right)+$ $\partial_{2}(\beta) f_{m-1}-\left(C_{m}^{2} \partial_{2}^{2}(\beta)+C_{m}^{1} \partial_{2}^{1}(\gamma)\right) f_{m}=0$, that is

$$
\partial_{1}\left(f_{m-1}\right)+\beta \partial_{2}\left(f_{m-1}\right)+\partial_{2}(\beta) f_{m-1}-\left(C_{m}^{2} \partial_{2}^{2}(\beta)+C_{m}^{1} \partial_{2}^{1}(\gamma)\right) f_{m}=0 .
$$

Evidently, $\left(f_{m-1} / f_{m}\right) \in K\left[X_{1}, X_{2}\right]$ since $f_{m} \in K \backslash\{0\}$ and, dividing the last equality by $f_{m}$, we obtain $P_{m}\left(f_{m-1} / f_{m}, \gamma\right)=0$. Our claim is then proved.

Now, if $\operatorname{deg}_{X_{2}}(\beta)=1$, write $\beta=a X_{2}+b$ with $a, b \in K\left[X_{1}\right], \operatorname{deg} a \geq 1$, and take $g \in K\left[X_{1}\right] \backslash\{0\}$ such that $\operatorname{deg}_{X_{1}}(g)<\operatorname{deg}_{X_{1}}(a)$. Let $m \geq 1$ be an integer and suppose that the equation $P_{m}\left(Z, g X_{2}\right)=0$ has a solution $f \in K\left[X_{1}, X_{2}\right]$ :

$$
\partial_{1}(f)+\left(a X_{2}+b\right) \partial_{2}(f)+a f-m g=0 .
$$

Clearly, we have $f \neq 0$. Write

$$
f=\sum_{i=0}^{r} f_{i} X_{2}^{i}, \text { with } f_{i} \in K\left[X_{1}\right] \quad \text { for every } i=0, \ldots, r, f_{r} \neq 0 .
$$

If $r \geq 1$, then looking at the coefficient of $X_{2}^{r}$ in equality (8), we obtain $f_{r}^{\prime}+r a f_{r}+a f_{r}=$ 0 . Hence $a(r+1) f_{r}=-f_{r}^{\prime}$, which is absurd, since $a \neq 0$ and $r+1 \neq 0$. If $r=0$, then by (8) we have $f_{0}^{\prime}+a f_{0}-m g=0$, which is also absurd since $\operatorname{deg}(g)<\operatorname{deg}(a)$ by hypothesis. Thus, for every integer $m \geq 1$, the equation $P_{m}\left(Z, g X_{2}\right)=0$ does not have any solution in $K\left[X_{1}, X_{2}\right]$. Hence the operator $d+g X_{2}$ does not admit any Darboux operator in $K\left[X_{1}, X_{2}\right]\left\langle\partial_{2}\right\rangle$ of order $m$ by the above claim. Then $A_{2} \cdot\left(d+g X_{2}\right)$ is a maximal left ideal of $A_{2}$ by Theorem 2.1 .

If $\operatorname{deg}_{X_{2}}(\beta) \geq 2$, take $g \in K\left[X_{1}\right]$ such that $g \notin-\left(\partial_{2}^{2}(\beta) / 2\right) \mathbb{N}$. Let $m \geq 1$ be an integer and suppose that the equation $P_{m}\left(Z, g X_{2}\right)=0$ has a solution $f \in K\left[X_{1}, X_{2}\right]$

$$
\partial_{1}(f)+\beta \partial_{2}(f)+\partial_{2}(\beta) f-(m(m-1) / 2) \partial_{2}^{2}(\beta)-m g=0 .
$$

This equality can also be written as

$$
\partial_{1}(f)+\partial_{2}(\beta f)-(m(m-1) / 2) \partial_{2}^{2}(\beta)-m g=0 .
$$

By the choice of $g$, we have $f \neq 0$. Then, the above equality is absurd: indeed, since $\operatorname{deg}_{X_{2}}(\beta) \geq 2$, the degree in $X_{2}$ of its left side is equal to $\operatorname{deg}_{X_{2}}(\beta)+\operatorname{deg}_{X_{2}}(f)-1 \geq$ 1. Thus, for every integer $m \geq 1$, the equation $P_{m}\left(Z, g X_{2}\right)=0$ does not have any solution in $K\left[X_{1}, X_{2}\right]$. Hence the operator $d+g X_{2}$ does not admit any Darboux operator in $K\left[X_{1}, X_{2}\right]\left\langle\partial_{2}\right\rangle$ of order $m$ by the above claim. Then $A_{2} \cdot\left(d+g X_{2}\right)$ is a maximal left ideal of $A_{2}$ by Theorem 2.1. 
ExAmple 2.7. Cyclic maximal left ideals of $A_{2}$.

The Main Theorem provides a powerful tool to produce explicit examples of cyclic maximal left ideals of $A_{2}$ : one considers a simple derivation of $K\left[X_{1}, X_{2}\right]$ of the type $d=$ $\partial_{1}+\beta \partial_{2}$ and then takes $A_{2} \cdot\left(d+g X_{2}\right)$ where $g \in K\left[X_{1}\right] \backslash\{0\}, g \notin-\left(\partial_{2}^{2}(\beta) / 2\right) \mathbb{N}$ when $\operatorname{deg}_{X_{2}}(\beta) \geq 2$ and $\operatorname{deg}_{X_{1}}(g)<\operatorname{deg}_{X_{1}}\left(\partial_{2}(\beta)\right)$ when $\operatorname{deg}_{X_{2}}(\beta)=1$.

Simple derivations of the type $d=\partial_{1}+\beta \partial_{2}$ with $\beta \in K\left[X_{1}, X_{2}\right]$ can be found in the literature. We give a few examples.

Case $\operatorname{deg}_{X_{2}}(\beta)=1$.

The best known example in this case is obtained by taking

$$
\beta=a X_{2}+b \quad \text { with } a, b \in K\left[X_{1}\right] \backslash\{0\}, \operatorname{deg}(a)>\operatorname{deg}(b) .
$$

The simplicity of $d$ is an immediate consequence of a theorem of Shamsuddin [7, Theorem 3.2.1, p. 151] or [3, Proposition 3.2, p. 410].

Other interesting "linear" examples can be obtained by using an algorithm that has been established in [7, p. 152-155]; for example, we get one of them by taking

$$
\beta=X_{1} X_{2}+\left(X_{1}^{3}+1\right) .
$$

Case $\operatorname{deg}_{X_{2}}(\beta)=2$.

Two important examples in this case are obtained by taking

(i) $\beta=X_{2}^{2}-p\left(X_{1}\right)$ with $p\left(X_{1}\right) \in K\left[X_{1}\right], \operatorname{deg}\left(p\left(X_{1}\right)\right)$ odd,

(ii) $\beta=X_{2}^{2}-\left(X_{1}^{2}-e\right)$ with $e \in K \backslash\{2 k+1 ; k \in \mathbb{Z}\}$.

The simplicity of such $d$ 's is proved in [6].

Other interesting "quadratic" examples are given in the same paper.

Case $\operatorname{deg}_{X_{2}}(\beta)=n, n \geq 2$ arbitrary.

One important example in this case is obtained by taking

$$
\beta=X_{2}^{n}+p X_{1} \text { with } p \in K \backslash\{0\} .
$$

The simplicity of $d$ is proved in [8].

Another example is given in [4].

if $\beta \in \mathbb{Q}\left[X_{1}, X_{2}\right]$ is an homogeneous polynomial of degree $n \geq 2$ that is irreducible over $\mathbb{Q}$, then $d:=\partial_{1}+\beta \partial_{2}$ is a simple derivation of $\mathbb{C}\left[X_{1}, X_{2}\right]$.

REMARK 2.8. (a) The family of examples of Coutinho (hence also the first family of examples of Stafford), given in the introduction can easily be obtained using our Main Theorem. Indeed, if $\beta=a X_{2}+b$ with $a, b \in K\left[X_{1}\right] \backslash\{0\}, \operatorname{deg}(a)>\operatorname{deg}(b)$, then $d:=\partial_{1}+\beta \partial_{2}$ is a simple derivation of $K\left[X_{1}, X_{2}\right]$ as was mentioned above. Then, if we take $g \in K\left[X_{1}\right] \backslash\{0\}$ with $\operatorname{deg}(g)<\operatorname{deg}(a)$, we can conclude that $A_{2} \cdot\left(d+g X_{2}\right)$ is a maximal left ideal of $A_{2}$.

(b) Similarly, the second family of examples of Stafford given in the introduction can also be obtained with our Main Theorem. Indeed, if $\beta=\lambda X_{2}^{2}+X_{1} X_{2}+1$ with $\lambda \in K \backslash \mathbb{Z}$, then by [6, p. 5106-5107 and Theorem 8.3 p. 5108], $d:=\partial_{1}+\beta \partial_{2}$ is a simple derivation of $K\left[X_{1}, X_{2}\right]$. Now, if we take $\gamma=v X_{2}$ with $v \in K\left[X_{1}\right] \backslash\{0\}, v / \lambda \notin-\mathbb{N}$, then $v \notin-\left(\partial_{2}^{2}(\beta) / 2\right) \mathbb{N}$ and we can conclude that $A_{2} \cdot\left(\partial_{1}+\left(\lambda X_{2}^{2}+X_{1} X_{2}+1\right) \partial_{2}+v X_{2}\right)$ is a maximal left ideal of $A_{2}$. Note that with our method, and contrary to what was done by Stafford, we need not suppose that $K$ is a field of infinite degree over $\mathbb{Q}$ (we only need to know that $K$ contains $\mathbb{Q}$ ), nor do we have to suppose that $\lambda \in K \backslash \mathbb{Q}$ (we only need to know that $\lambda \in K \backslash \mathbb{Z}$ ). 
ACKNOWLEDGEMENT. We are grateful to the referee who made several suggestions that improved the presentation of this paper.

\section{REFERENCES}

1. J. Berstein and V. Lunts, On non-holonomic irreducible D-modules, Invent. Math. 94 (1988), 223-243.

2. G. Bratti and M. Takagi, Differential equations and maximal ideals on the Weyl algebra $A_{2}(\mathbb{C})$, Rend. Sem. Mat. Univ. Padova 107 (2002), 209-223.

3. S. C. Coutinho, d-simple rings and simple D-modules, Math. Proc. Camb. Phil. Soc. 125 (1999), 405-415.

4. S. C. Coutinho, An example of differential simplicity, preprint.

5. Y. Lequain, D. Levcovitz and J. C. Souza Jr., d-simple rings and principal maximal ideals of the Weyl algebra, Glasgow Math. J. 47 (2005), 269-285.

6. A. Maciejewski, J. Moulin-Ollagnier and A. Nowicki, Simple quadratic derivations in two variables, Comm. Algebra 29 (2001), 5095-5113.

7. A. Nowicki, Polynomial derivations and their rings of constants (Nicholas Copernicus University Press, Torun, 1994).

8. A. Nowicki, An example of simple derivation in two variables, preprint. $22-42$

9. A. Seidenberg, Differential ideals of finitely generated type, Amer. J. Math. 89 (1967),

10. J. T. Stafford, Non-holonomic modules over Weyl algebras, Invent. Math. 79 (1985), $619-638$. 